\title{
Signal Acquisition Using Surface EMG and Circuit Design Considerations for Robotic Prosthesis
}

\author{
Muhammad Zahak Jamal
}

Additional information is available at the end of the chapter

http://dx.doi.org/10.5772/52556

\section{Introduction}

Electromyography (EMG) is the subject which deals with the detection, analysis and utilization of electrical signals emanating from skeletal muscles. The field of electromyography is studied in Biomedical Engineering. And prosthesis using electromyography is achieved under Biomechatronics [1]. The electric signal produced during muscle activation, known as the myoelectric signal, is produced from small electrical currents generated by the exchange of ions across the muscle membranes and detected with the help of electrodes. Electromyography is used to evaluate and record the electrical activity produced by muscles of a human body. The instrument from which we obtain the EMG signal is known as electromyograph and the resultant record obtained is known as electromyogram [2].

The human body is a wonder of nature. The functioning of human body is an intriguing and fascinating activity. Motion of the human body is a perfect integration of the brain, nervous system and muscles. It is altogether a well-organized effort of the brain with 28 major muscles to control the trunk and limb joints to produce forces needed to counter gravity and propel the body forward with minimum amount of energy expenditure [3]. The movement of the human body is possible through muscles in coordination with the brain. Whenever the muscles of the body are to be recruited for a certain activity, the brain sends excitation signals through the Central Nervous System (CNS). Muscles are innervated in groups called 'Motor Units'. A motor unit is the junction point where the motor neuron and the muscle fibers meet. A depiction of the Motor Unit is given in Figure 1. When the motor unit is activated, it produces a 'Motor Unit Action Potential' (MUAP) [4]. The activation from the Central Nervous System is repeated continuously for as long as the muscle is required to generate force. This continued activation produces motor unit action potential trains. The trains from concurrently active motor units superimpose to produce the resultant EMG 
signal. A group of muscles are involved in a certain movement of the human body. The number of muscles recruited depends upon the activity in which the body is involved. E.g. in lifting a small weight such as a tiny pebble, fewer amount of muscles will be involved as compared to lifting a heavy mass like a $6 \mathrm{~kg}$ weight, where the muscles employed will be greater. In technical terms, whenever it is required to generate greater force, the excitation from the Central Nervous System increases, more motor units are activated and the firing rate of all the motor units increase resulting in high EMG signal amplitudes [4,5].

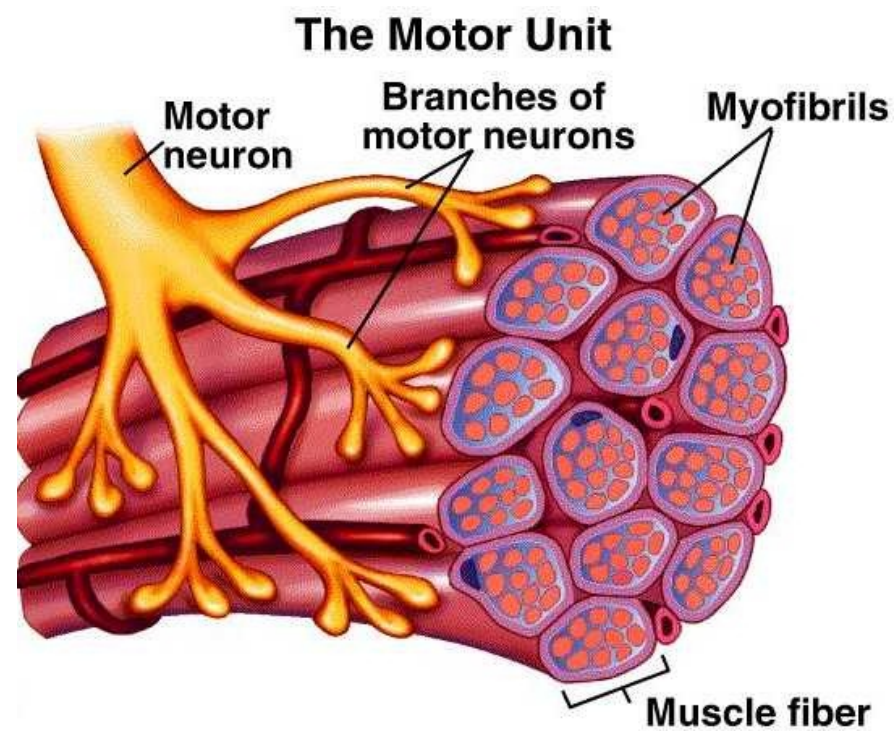

Figure 1. A Motor Unit consists of one motor neuron and all the muscle fibers it stimulates [6]

Electromyography enables us to generate force, create movements and allow us to do countless other functions through which we can interact with the world around us. The electromyograph is a bioelectric signal which has, over the years, developed a vast range of applications. Clinically, electromyography is being used as diagnostic tool for neurological disorders. It is frequently being used for assessment of patients with neuromuscular diseases, low back pain and disorders of motor control [7]. Other than physiological and biomechanical research, EMG has been developed as an evaluation tool in applied research, physiotherapy, rehabilitation, sports medicine and training, biofeedback and ergonomics research.

In the recent past, EMG has also found its use in rehabilitation of patients with amputations in the form of robotic prosthesis. EMG proves to be a valuable tool as it provides a natural way of sensing and classifying different movements of the body. A multi-degree of freedom robotic mechanism can effectively imitate the motion of the human limb. Recent advances in electronics and microcontroller technology have allowed improved control options for robotic mechanisms. One of the most vital advantages of microprocessor technology in robotic prosthetics is the advanced EMG filtering algorithms. Nowadays, control options are even available to those who were not at one time qualified for such prosthetic management. 
This chapter will discuss in detail, the effective use of surface electromyography (SEMG) as a tool for achieving robotic prosthesis. An elaborate account of SEMG electrode types, signal acquisition technique, electronics circuit design considerations and the control procedure to drive electric motors in a robotic mechanism is provided in this chapter.

\section{EMG electrodes and its types}

The bioelectrical activity inside the muscle of a human body is detected with the help of EMG electrodes. There are two main types of EMG electrodes: surface (or skin electrodes) and inserted electrodes. Inserted electrodes have further two types: needle and fine wire electrodes. The three electrodes (needle, fine wire and surface) are explained as follows. Among these three electrodes, surface EMG electrodes will be specifically discussed in detail as it pertains to the topic of this chapter.

\subsection{Needle electrodes}

Needle electrodes are widely used in clinical procedures in neuromuscular evaluations. The tip of the needle electrode is bare and used as a detection surface. It contains an insulated wire in the cannula. The signal quality from the needle electrodes is comparatively improved from other available types. Needle electrodes have two main advantages. One is that its relatively small pickup area enables the electrode to detect individual MUAPs during relatively low force contractions. The other is that the electrodes may be conveniently repositioned within the muscle (after insertion) so that new tissue territories may be explored [5]. A needle electrode is shown in Figure 2.

\section{Concentric Needle Electrodes}

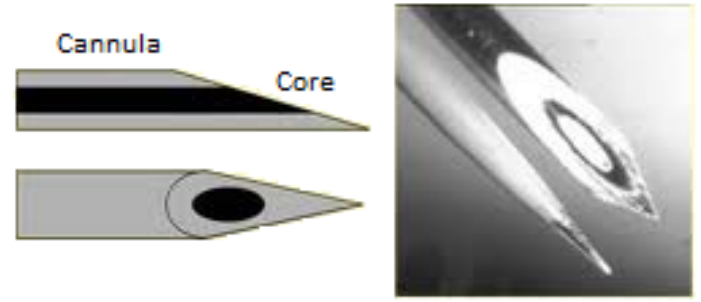

Figure 2. A Needle EMG Electrode [8]

\subsection{Fine wire electrodes}

Wire electrodes are made from any small diameter, highly non-oxidizing, stiff wire with insulation. Alloys of platinum, silver, nickel, and chromium are typically used. Wire electrodes are extremely fine, they are easily implanted and withdrawn from skeletal muscles, and they are generally less painful than needle electrodes whose cannula remains 
inserted in the muscle throughout the duration of the test [5]. A fine wire electrode is shown in Figure 3.

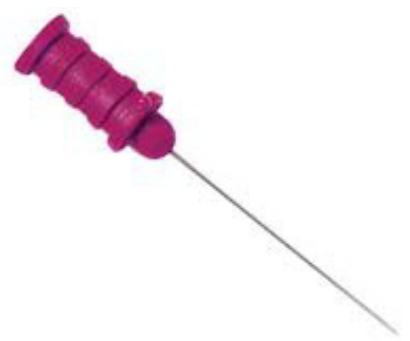

Figure 3. Fine Wire EMG Electrode

\subsection{Surface EMG electrode}

Surface EMG electrodes provide a non-invasive technique for measurement and detection of EMG signal. The theory behind these electrodes is that they form a chemical equilibrium between the detecting surface and the skin of the body through electrolytic conduction, so that current can flow into the electrode.

These electrodes are simple and very easy to implement. Application of needle and fine wire electrodes require strict medical supervision and certification. Surface EMG electrodes require no such formalities. Surface EMG electrodes have found their use in motor behavior studies, neuromuscular recordings, sports medical evaluations [9] and for subjects who object to needle insertions such as children. Apart from all this, surface EMG is being increasingly used to detect muscle activity in order to control device extensions to achieve prosthesis for physically disabled and amputated population.

Surface EMG has some limitations as well. Since these electrodes are applied on the skin, hence, they are generally used for superficial muscles only. Crosstalk from other muscles is a major problem. Their position must be kept stable with the skin; otherwise, the signal is distorted.

\subsubsection{Types of EMG Electrodes}

There are two types of surface EMG electrodes: Gelled and Dry EMG electrodes [10].

\subsubsection{Gelled EMG Electrodes}

Gelled EMG electrodes contain a gelled electrolytic substance as an interface between skin and electrodes. Oxidation and reduction reactions take place at the metal electrode junction. Silver - silver chloride $(\mathrm{Ag}-\mathrm{AgCl})$ is the most common composite for the metallic part of gelled electrodes. The $\mathrm{AgCl}$ layer allows current from the muscle to pass more freely across the junction between the electrolyte and the electrode. This introduces less electrical noise into the measurement, as compared with equivalent metallic electrodes (e.g. Ag). Due to this fact, $\mathrm{Ag}-\mathrm{AgCl}$ electrodes are used in over $80 \%$ of surface EMG applications [10]. 
Disposable gelled EMG electrodes are most common; however, reusable gelled electrodes are also available. Special skin preparations and precautions such as (hair removal, proper gel concentration, prevention of sweat accumulation etc.) are required for gelled electrodes in order to acquire the best possible signal. Gelled EMG electrodes are shown in Figure 4.

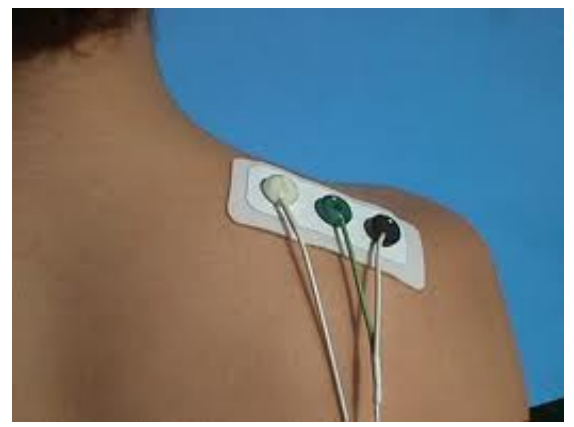

Figure 4. Gelled EMG Electrodes

\subsubsection{Dry EMG electrodes}

Dry EMG electrodes do not require a gel interface between skin and the detecting surface. Bar electrodes and array electrodes are examples of dry electrodes. These electrodes may contain more than one detecting surface. In many examples, an in-house pre-amplification circuitry may also be employed in these electrodes. A reusable bar electrode is shown in Figure 5. Dry electrodes are usually heavier $(>20 \mathrm{~g})$ as compared to gelled electrodes $(<1 \mathrm{~g})$. This increased inertial mass can cause problems for electrode fixation; therefore, a material for stability of the electrode with the skin is required [10].

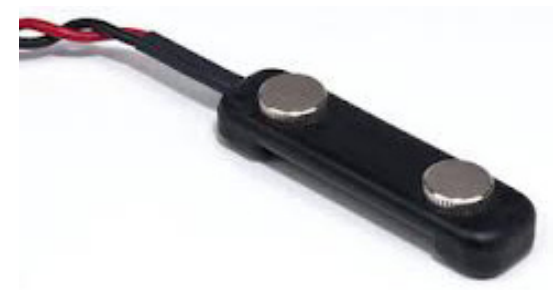

Figure 5. A Reusable Bar Electrode (an Example of Dry EMG Electrode)

\subsubsection{Categories of Surface EMG Electrodes}

There are two categories of surface EMG electrodes [5]: Passive and Active EMG electrodes. They are briefly explained as follows:-

\subsubsection{Passive EMG electrodes}

These electrodes should be connected to an external amplification circuitry with the help of connecting wires for the proper acquisition of the EMG signal. Passive EMG electrodes can be disposable or reusable. 
Electrodes shown in Figure 4 and Figure 5 both fall under passive surface EMG electrodes.

\subsubsection{Active EMG electrodes}

Active EMG electrodes contain a pre-amplifier attachment for surface electrodes. Needle and fine wire surface electrodes are also available. These electrodes usually fall under the dry surface EMG electrodes type. The in-house high impedance amplifier in these electrodes transfers the pre-amplified signal to the rest of the circuitry. Figure 6 shows an active EMG electrode.

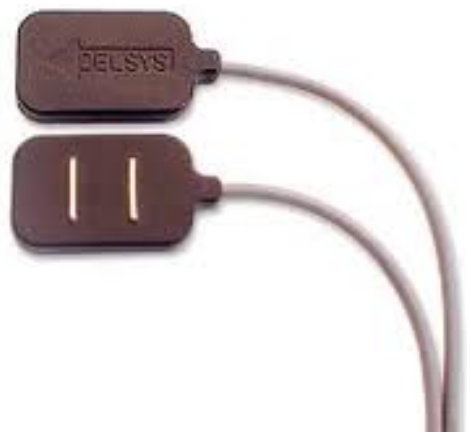

Figure 6. The Delsys 2.1 Active EMG Electrode [11]

\section{EMG electrode placement and signal acquisition technique}

Surface EMG is relatively easy to use as compared to other EMG electrodes. This is the reason why it is being extensively used in the control of robotic mechanisms to achieve prosthesis. It is also widely used in latest EMG researches by engineers as no medical certification or expertise is required for its application. Its use in rehabilitation prosthesis is favorable as it does not cause any kind of discomfort to the subject on whom it is applied. Other kinds of EMG electrodes (needle and fine wire), when inserted into the skin of the subject, may effect a twitching sensation and cause him or her to make movements.

In order to get the best results from SEMG, it is really important to have a proper understanding of the muscles from which the EMG signal is being extracted. The placement on skin also requires adequate study and requires skin preparation beforehand as well.

The EMG electrodes, their types, sub-types and categories have already been explained in detail in the previous section. Since, our concern is only with Surface EMG (SEMG), hence, we will only deal with the placement and signal acquisition technique using surface EMG electrodes.

\subsection{Overview of muscle architecture}

Skeletal muscle architecture is defined in terms of "the arrangement of muscle fibers relative to the axis of force generation." The skeletal muscle arrangement as well as their activity 
reveals striking organization at the macroscopic level. The functional properties of the skeletal muscle depend strongly on their architecture [12].

There are various kinds of muscle fiber arrangements, which are discussed as follows:-

i. Muscles with fibers that extend parallel to the muscle force-generating axis are termed parallel, fusiform or longitudinally arranged muscles. Examples of such types of muscles are Biceps Brachii (bicep muscle) and Sartorius (groin muscle).

ii. Muscles with fibers that are oriented at a single angle relative to the force generating axis are termed unipennate muscles. Example of unipennate muscle is Extensor Digitorum Longus.

iii. The angle between the fiber and the force-generating axis generally varies from $0^{\circ}$ to $30^{\circ}$. The muscles are oriented at more than one angle. Most muscles fall into this category and they are called as multipennate muscles. Examples are Rectus Femoris which is bipennate and Deltoid which is multipennate.

iv. The muscles which surround an opening so as to form a closed shape are known as circular muscles. Example of such kind of muscle is Orbicularis Oris (mouth muscle).

v. The muscles in which their fibers converge on the insertion to maximize force of contraction are known as Convergent muscles. E.g. Pectoralis Major.

A detailed depiction of these muscle arrangements is provided in Figure 7.
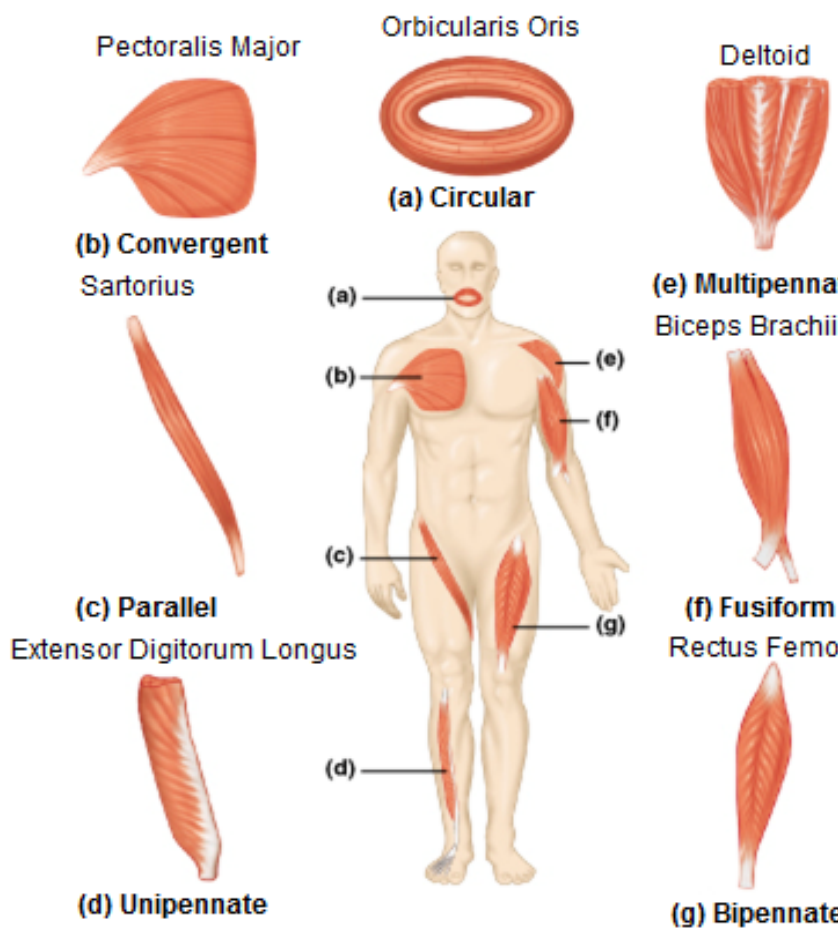

(e) Multipennate

Biceps Brachii

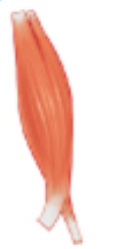

(f) Fusiform

Rectus Femoris

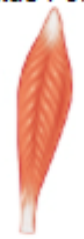

(g) Bipennate

Figure 7. Muscles and their Architecture [13] 


\subsection{Skin preparation}

Application of surface EMG electrodes requires proper skin preparation beforehand. In order to obtain a good quality EMG signal, the skin's impedance must be considerably reduced. For this purpose, the dead cells on the skin e.g. hair must be completely removed from the location where the EMG electrodes are to be placed. It is advisable to use an abrasive gel to reduce the dry layer of the skin [9]. There should be no moisture on the skin. The skin should be cleaned with alcohol in order to eliminate any wetness or sweat on the skin.

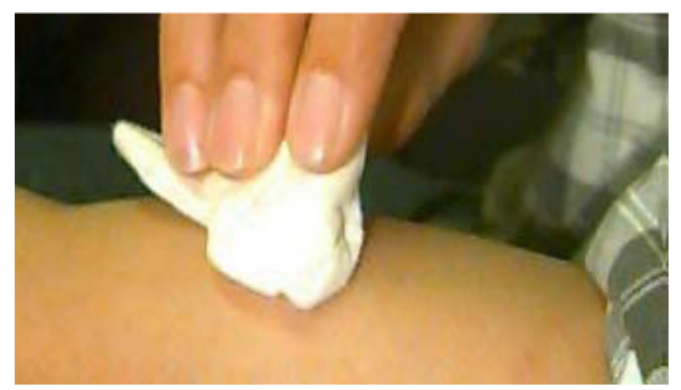

Figure 8. Skin Preparation prior to application of EMG electrodes

\subsection{EMG electrode placement}

The application of EMG electrodes requires adequate know how of the skeletal muscles. The EMG electrode placement will be discussed in detail under this section.

In most cases, two detecting surfaces (or EMG electrodes) are placed on the skin in bipolar configuration $[14,15]$. In order to acquire the best possible signal, the EMG electrode should be placed at a proper location and its orientation across the muscle is important. The surface EMG electrodes should be placed between the motor unit and the tendinous insertion of the muscle, along the longitudinal midline of the muscle [15]. The distance between the center of the electrodes or detecting surfaces should only be $1-2 \mathrm{~cm}$. The longitudinal axis of the electrodes (which passes through both detecting surfaces) should be parallel to the length of the muscle fibers.

As mentioned previously, the EMG detecting surfaces should be placed in between the motor unit and the tendon insertion of the muscle. Detecting surfaces placed on the belly of the muscle has proved to be a more than acceptable location. Here, the target muscle fiber density is the highest [15]. Figure 9 shows the proper EMG electrode placement. When the electrodes are arranged in this way, the detecting surfaces intersect most of the same muscle fibers, and as a result, an improved superimposed signal is observed.

The electrodes should not be placed elsewhere. In the past, a misconception prevailed that the EMG detecting surfaces should be placed on the motor unit. But, as a matter of fact, the electrode location on the motor point serves as the worst location for signal detection [15]. 
Similarly, the electrodes should neither be placed at or near the tendon nor at the edge of the muscle. The muscle fibers become thinner and smaller in number when they approach the tendon of the muscle resulting in a weak EMG signal, proving the fact that electrode placement near the tendon is not feasible. If the electrode is placed at the edge of the muscle, the chances of crosstalk from other muscles will considerably increase, and the resultant signal will be disturbed by those of other muscles [15].

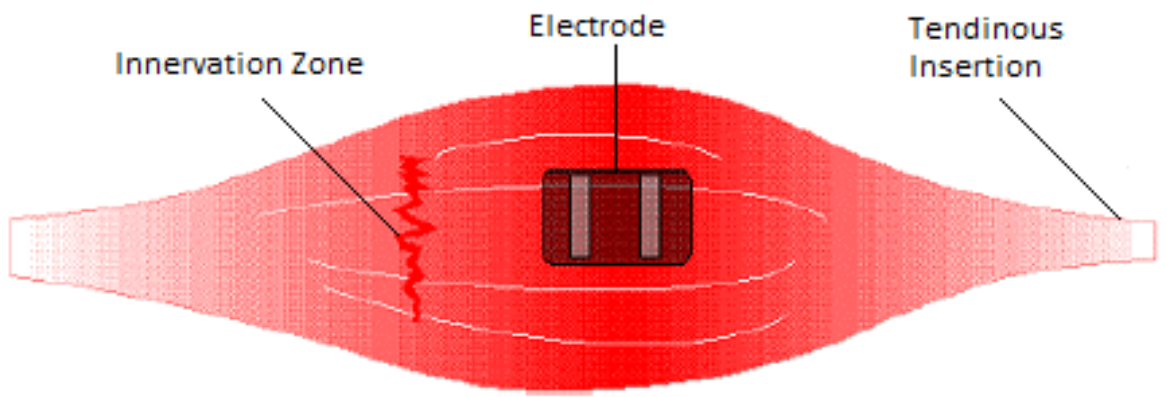

Figure 9. The ideal position of the electrode (two detecting surfaces) is between the innervation zone (or motor unit) and the tendinous insertion (or belly of the muscle) [15]

\subsection{General concerns}

Before we move on to the signal acquisition phase, it is very important to get acquainted with the EMG signal and the various concerns and factors affecting the qualitative properties of the signal.

The EMG signal's amplitude lies in between 1-10 mV, making it a considerably weak signal. The signal lies in the frequency range from $0-500 \mathrm{~Hz}$ and most dominant in between $50-150$ $\mathrm{Hz}$ [15].

The EMG signal is highly influenced by noise [16], as shown in Figure 10. The characteristics of electrical noise can be caused from various sources. Ambient noise can be caused by electromagnetic radiation sources e.g. radio transmission devices, fluorescent lights and power line interference from electrical wires. These interferences are almost impossible to avoid from external means. This particular noise exists in the frequency range of 50-60 Hz. Noise can also be generated from motion artifact. The two main sources of this noise are instability of electrode skin interface and movement of the electrode cable and lies mostly in the range of 0 $20 \mathrm{~Hz}$. It can be eliminated by proper set of EMG equipment and circuitry. The maximum fidelity of the signal is determined by the acquired EMG signal-to-noise ratio $[5,14]$.

\subsection{Reference electrode placement}

The signal from the EMG detecting surfaces is gathered with respect to a reference. An EMG reference electrode acts as a ground for this signal. It should be placed far from the EMG detecting surfaces, on an electrically neutral tissue [15]. 


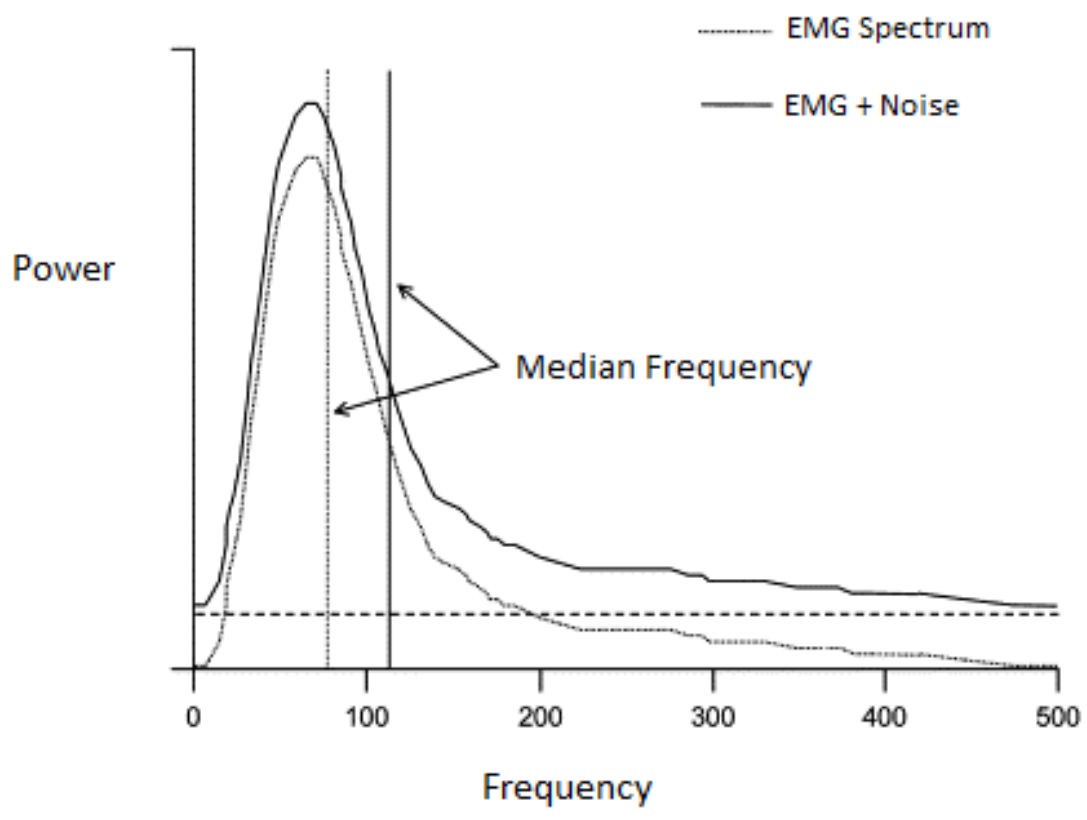

Figure 10. EMG Spectrum and noise influence on this spectrum [16]

\subsection{EMG signal acquisition circuitry and configurations}

The EMG electrode placement has been elaborately explained under the previous heading. So, after properly understanding the target muscle profile, preparing the skin and positioning the EMG detecting surfaces, comes the EMG signal acquisition step.

EMG signal is acquired through differential amplification technique. The differential amplifier should have high input impedance and very low output impedance. Ideally, a differential amplifier has infinite input and zero output impedance [17].

Differential amplification is achieved with the help of an instrumentation amplifier for high input impedance. A classic three amplifier instrumentation amplifier is shown in Figure 11.

The instrumentation amplifier carries out differential amplification by subtracting the voltages $V_{1}$ and $V_{2}$. This way, the noise signal which is common at $V_{1}$ and $V_{2}$ (electrode inputs) e.g. power line interference etc. are eliminated. The tendency of a differential amplification to reject signals common to both inputs is determined by common mode rejection ratio (CMRR). A CMRR of $90 \mathrm{~dB}$ is enough for elimination of common signals for instrumentation amplifiers, but latest technology, even though expensive, provides us with a CMRR of $120 \mathrm{~dB}$. But there are reasons for not pushing the CMRR to the limit, as the electrical noise detected by the electrodes may not be in phase [15]. The gain for the instrumentation amplifier can be set using a single resistor ( $\left.R_{\text {gain }}\right)$. The gain equation and output equation of the instrumentation amplifier is given in Eq. 1 and 2. 


$$
\begin{aligned}
& \text { Gain }=\left(1+\frac{2 R 1}{\text { Rgain }}\right) \frac{R 3}{R 2} \\
& \text { Vout }=(V 2-V 1) \times \text { Gain }
\end{aligned}
$$

A small gain of 5 or 6 is recommended for signal acquisition. Extensive amplification will be carried out in further steps. The placement of the EMG detecting surfaces can be done through three different configurations: monopolar, bipolar and multipolar.

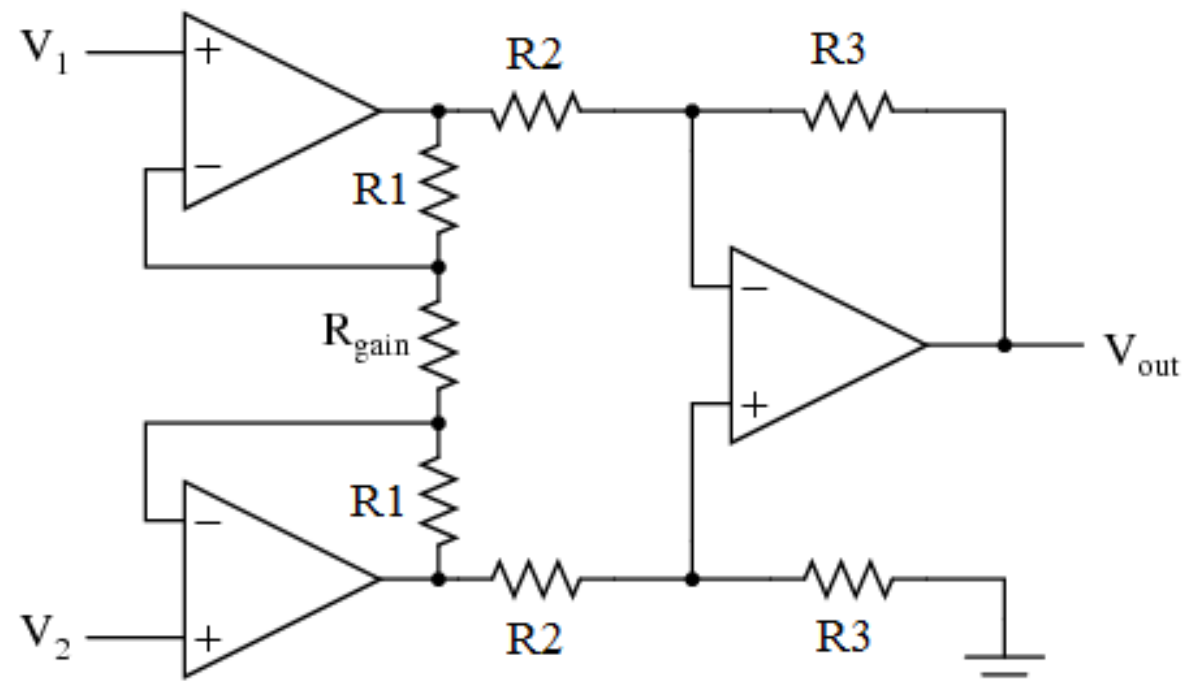

Figure 11. A Three Amplifier Instrumentation Amplifier

\subsubsection{Monopolar configuration}

The monopolar configuration is implemented using only a single electrode on the skin with respect to a reference electrode as shown in Figure 12. This method is used because of its simplicity, but is strictly not recommended as it detects all the electrical signals in the vicinity of the detecting surface $[5,14]$.

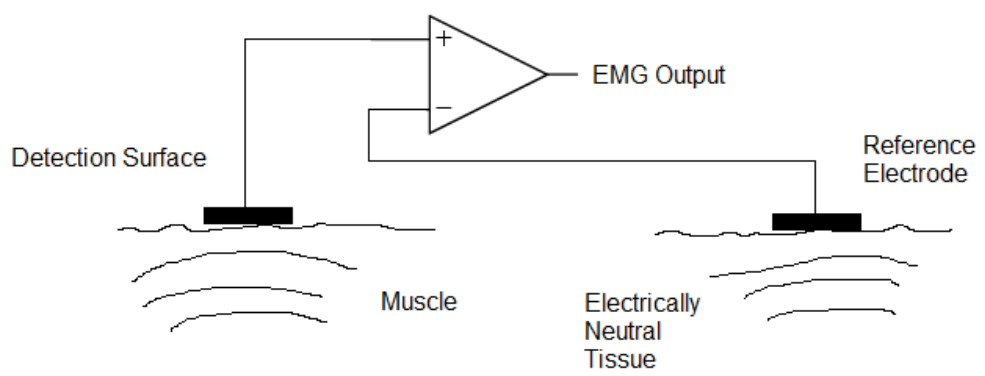

Figure 12. Monopolar signal acquisition technique 


\subsubsection{Bipolar configuration}

Bipolar configuration is used to acquire EMG signal using two EMG detecting surfaces with the help of a reference electrode. The signals from the two EMG surfaces are connected to a differential amplifier. The two detecting surfaces are placed only 1-2 cm from each other. The differential amplifier suppresses the common noise signals to both inputs and then amplifies the difference $[5,14]$. The limitations of the monopolar configuration are catered for by this configuration. This is the most commonly used electrode configuration. The bipolar EMG electrode configuration is shown is Figure 13.

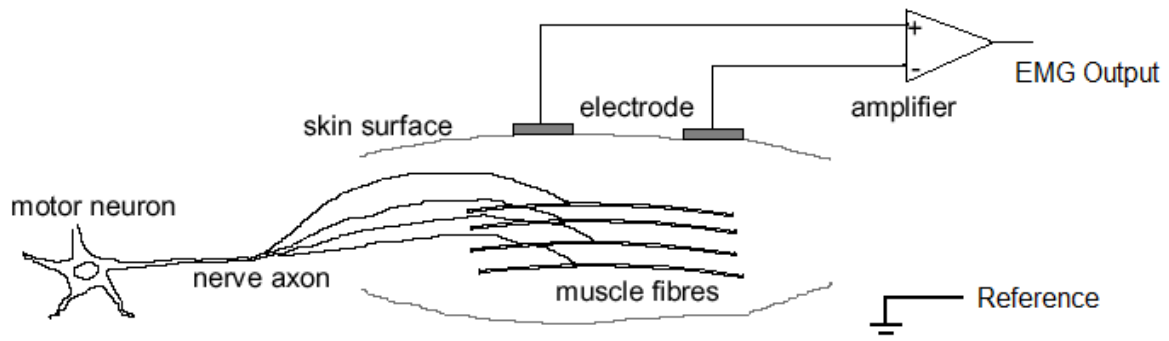

Figure 13. Bipolar Configuration

\subsubsection{Multipolar configurations}

This configuration uses more than two detecting surfaces to acquire the EMG signal with the help of a reference electrode. This configuration further reduces crosstalk and noise concerns [14]. A much more enhanced EMG signal is acquired from this configuration. The signals from three or more EMG detecting surfaces, placed $1-2 \mathrm{~cm}$ from each other, are passed through more than two stages of differential amplification. For example if three detecting surfaces are used then double differential technique is employed.

This configuration is used in comprehensive researches carried out to study EMG muscle fiber orientation, conduction velocity and motor point localization.

\section{Electrical design considerations}

This section will discuss the electrical design considerations in order to synthesize the best possible EMG signal from the muscles of the human body in thorough detail. The basic circuitry for signal acquisition or preamplification circuitry is explained in due detail in the previous section. In this section we will discuss the circuitry implemented after the preamplification stage.

\subsection{Filtering}

As discussed earlier, there are many concerns regarding the proper detection of the EMG signal. Once the electrode is properly placed and the signal is extracted, noise plays a major 
role in hampering the recording of the EMG signal. For this purpose, the signal has to be properly filtered, even after differential amplification [18, 19].

The noise frequencies contaminating the raw EMG signal can be high as well as low. Low frequency noise can be caused from amplifier DC offsets, sensor drift on skin and temperature fluctuations and can be removed using a high pass filter. High frequency noise can be caused from nerve conduction and high frequency interference from radio broadcasts, computers, cellular phones etc. and can be deleted using a low pass filter.

In order to remove these high and low frequencies, high pass and low pass bio-filters will be discussed in adequate detail in this section.

\subsubsection{High pass filter}

A high pass filter is used to remove low frequency component from a particular electrical signal. A term 'cut-off frequency', denoted by ' $f_{c}$ ', is the frequency below which all frequencies are eliminated. All frequencies above $f_{c}$ are carried forward. The frequency range where the filter response is ' 1 ' and the signals are transmitted is known as 'passband' region. On the contrary, the frequency range where the filter response is ' 0 ' and the signals are attenuated is known as 'stop band' region [18]. A high pass filter response is shown in Figure 14 .

\section{High Pass Filter}

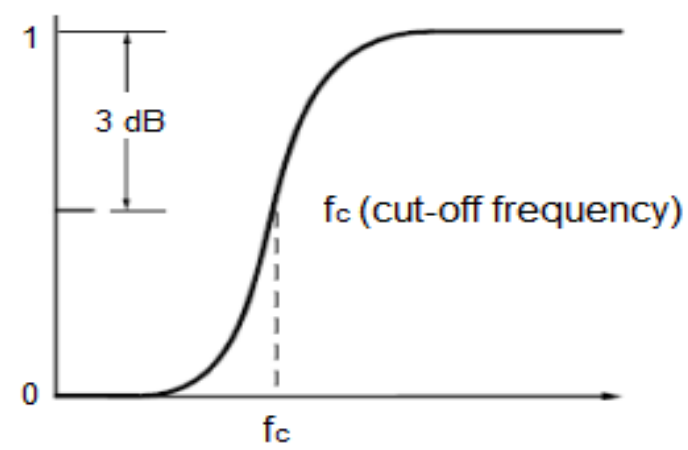

Figure 14. A high pass filter response

A high pass filter can be developed by using a resistor and a capacitor. This circuit will then be known as a CR circuit [20]. This circuit is a first order high pass filter. It is the simplest high pass filter possible. The high pass filtered signal is gathered across the resistor. The filter is shown in Figure 15.

The cut-off frequency of the high pass filter is given in Eq. 3.

$$
f c=\frac{1}{2 \pi R C}
$$


A second order high pass filter can also be designed. An effective design can employ an active electronic component [20]. The design uses two first order filters in series and is facilitated by an operational amplifier. The circuit is given Figure 16.

For this circuit, if $\mathrm{R}_{1}=\mathrm{R}_{2} ; \mathrm{C}_{1}=\mathrm{C}_{2}$ then $f_{c}$ is given as:-

$$
f c=\frac{1}{2 \pi R C}
$$

$R_{3}$ and $R_{4}$ are optional and are required for separate gain settings as:-

$$
\mathrm{A}_{0}=1+\mathrm{R}_{4} / \mathrm{R}_{3}
$$

Using a $2^{\text {nd }}$ order filter is recommended as they provide a roll-off of $40 \mathrm{~dB} / \mathrm{dec}$ as compared to $20 \mathrm{~dB} / \mathrm{dec}$ provided by $1^{\text {st }}$ order filters [18]. The use of active components can isolate the filter from the rest of the circuitry.

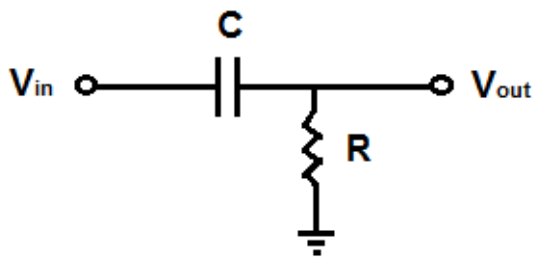

Figure 15. First order high pass filter

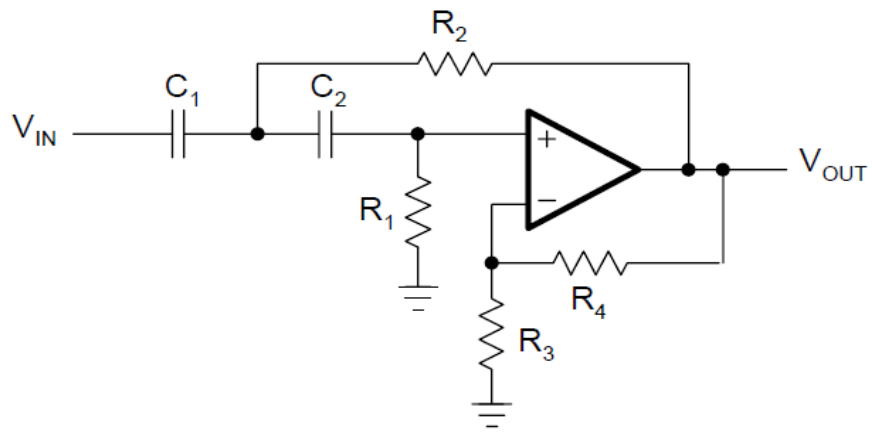

Figure 16. A $2^{\text {nd }}$ Order High Pass Filter

\subsubsection{Low pass filters}

The concept of low pass filters is entirely opposite to that of high pass filters. In these filters, the frequencies less than the cut-off frequency are transmitted and above that are removed [18]. A low pass filter response is shown in Figure 17.

The simplest low pass filter can be designed with the help of a resistor and a capacitor called as a $1^{\text {st }}$ order RC circuit [20]. The low pass filtered signal is detected across the capacitor. The $1^{\text {st }}$ order low pass filter circuit is shown in Figure 18. 


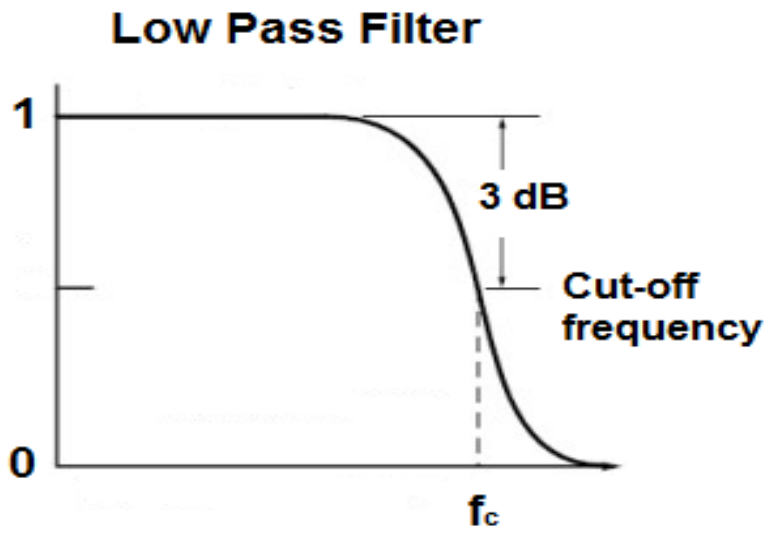

Figure 17. Low Pass Filter Response

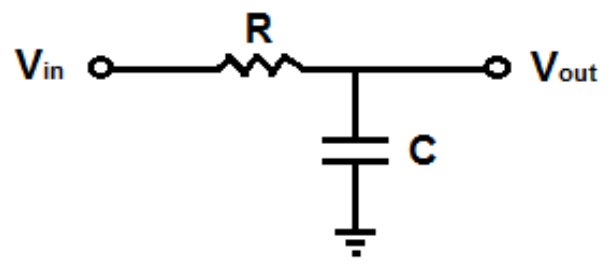

Figure 18. $1^{\text {st }}$ Order Low Pass Filter

The cut-off frequency equation for the circuit in Figure 18 is the same as that of Eq. 3.

A $2^{\text {nd }}$ order low pass filter can be more effective as compared to a $1^{\text {st }}$ order one. It can be designed by cascading two $1^{\text {st }}$ order filters attached to an operational amplifier. The circuit is given in Figure 19.

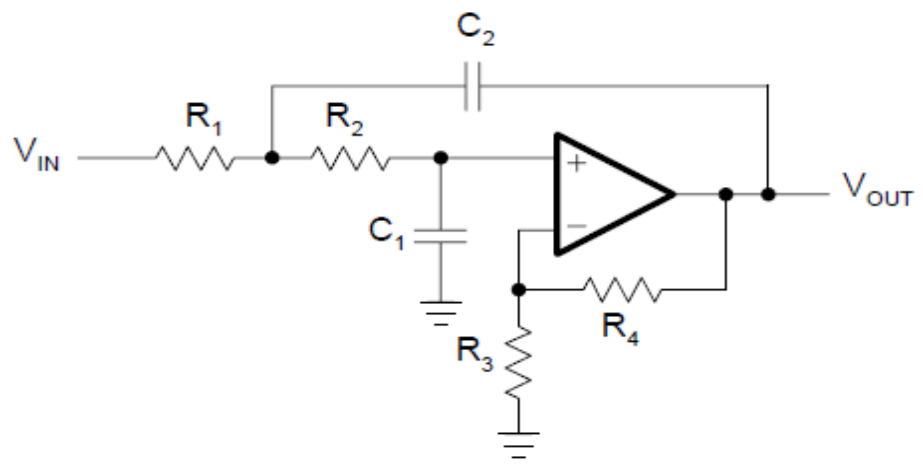

Figure 19. $2^{\text {nd }}$ Order Low Pass Filter

For $R_{1}=R_{2}$ and $C_{1}=C_{2}$, the cut-off frequency of the circuit in Figure 19 is the same as that of Eq. 4 . $R_{3}$ and $R_{4}$ are optional as they are required for separate gain settings as given in Eq. 5 . 
A $2^{\text {nd }}$ order low pass filter is again recommended as compared to a $1^{\text {st }}$ order one for the same reasons mentioned for a $2^{\text {nd }}$ order high pass filter.

\subsubsection{Band pass filtering for $E M G$}

As mentioned previously, for the transmission of pure EMG, the high and low frequency noise should be deleted. For this purpose, only a specific band of frequency should be carried forward [20]. This can be made possible with the help of a band pass filter. A band pass filter response is shown in Figure 20.

\section{Band Pass Filter}

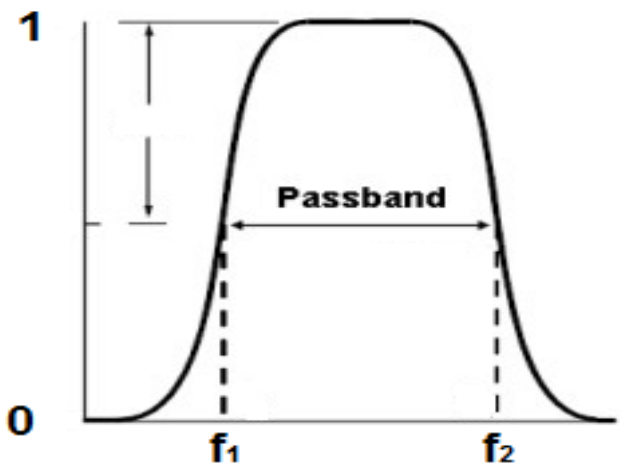

Figure 20. Band Pass Filter Response

The frequency region where the response of the EMG signal is ' 1 ' is called the 'passband' and in the case of band pass filter, it is between $f_{1}$ and $f_{2}$.

A band pass filter can be designed by connecting a low pass and a high pass filter in series. By selecting proper values of $\mathrm{R}$ and $\mathrm{C}$, we can develop a band pass filter which can carry forward the most effective component of the EMG signal. It is recommended that for EMG, $f_{1}$ should be $65-70 \mathrm{~Hz}$ and $f_{2}$ be $150-180 \mathrm{~Hz}$.

\subsection{Amplification}

After the signal has been filtered properly and a suitable band of EMG frequency is obtained, the next stage is amplification. The EMG signal obtained has to be powered up to a suitable level. The amplification of the EMG signal can be easily carried out with the help of a non-inverting amplifier, shown in Figure 21.

The gain of the amplifier is provided in the figure as ' $\mathrm{A}_{\mathrm{v}}$ '. The non-inverting amplifier is only used when the signal is being received from a single wire referenced to ground. Amplification can be done in stages in order to cater for chip requirements, by cascading them in series. 
The EMG signal, as mentioned before, is very weak i.e. only 1-10 $\mathrm{mV}$. For certain muscles, for which the signal response is very strong e.g. Biceps Brachii, a gain of 500-1000 can be enough. But for muscles, whose EMG response is weak e.g. Flexor Palmaris Longus (ring finger muscle), the gain settings should be very high i.e. 10000 .

The proper gain setting solely depends upon the signal response observed from the subject's target muscle. It is to be noted that every subject gives a separate signal response. Some subjects will give weak responses as compared to others. So, in that case, appropriate gain value should be set once the subject's EMG signal response is properly observed.

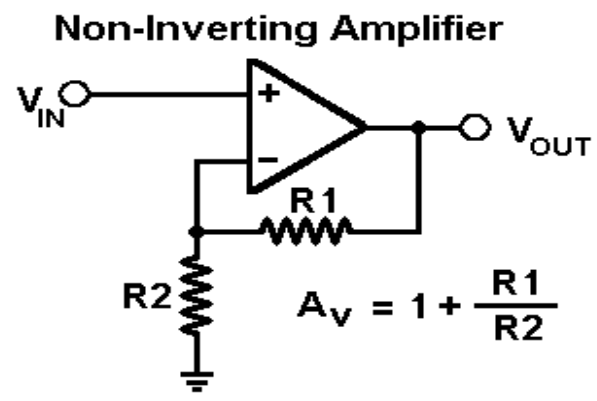

Figure 21. A Non-Inverting Amplifier

\section{Control technique}

In order to successfully achieve robotic prosthesis, an effective control technique is very important in order to drive the electric motors in the mechanism. With the advent of modern microcontroller technology, the control options available today have never been so effective.

For implementing the desired control to the motors, the amplified EMG signal in analog form has to be converted into digital format. After this, the motors are driven with the help of a microcontroller through the thresholding technique. These techniques will be discussed in detail in this section.

\subsection{Analog to digital conversion}

The digitization process of the analog signal is carried out with an Analog to Digital Converter (ADC). Nowadays, the ADC has become a common component of modern electronic devices. Their use has become highly varied and widespread. Before using the ADC, its specifications, advantages and limitations have to be analyzed in order to select the most appropriate one for the application. In the same way, important considerations have to be taken into account while converting EMG signals into digital format.

Control of the motor will be developed after the EMG signal is converted into digital format. A particular $\mathrm{ADC}$ has a specific range of conversion i.e. there are maximum and minimum levels defined for an ADC over which it can operate. An ADC can convert the analog signal over a certain number of bits. The number of bits which an ADC can convert is known as its 
"quantization scheme". If an ADC has a defined range and a quantization scheme of ' $n$-bits', then the resolution of the ADC can be given as:-

$$
\mathrm{V}_{\text {resolution }}=\mathrm{V}_{\text {range }} /(2)^{\mathrm{n}}
$$

While converting an EMG signal into digital format, three specifications should be taken into account. 1) Quantization, 2) Range of conversion and 3) Sampling rate [21].

The number of bits, which an analog signal can be converted into digital format by an ADC, is known as quantization. The maximum amount of voltage an ADC can convert into digital quantized bits defines the range of an ADC. The sampling rate means the number of samples an ADC can convert in one second.

After the EMG signal has been amplified up to a suitable level, the range of an ADC should be selected so that it can comprehend a particular voltage level. The number of quantization bits is important, as they determine the resolution of the ADC. The more the number of quantization bits, the less will be resolution of the ADC; the more it will help in control purposes. The ADC sampling rate is also a key consideration. It should be kept as large as possible so that the data loss of EMG is kept at a minimum [21].

ADCs are now available as a peripheral with microcontroller chips and can give sampling rates greater than $1000 \mathrm{kSPS}$ and quantization schemes of more than 24 bits.

\subsection{Thresholding and motor drive}

The control of robotic prosthesis is provided through the thresholding technique [21]. Once the signal is received in digital format, taking all necessary considerations as described before, a suitable threshold is applied to that particular quantized digital signal.

Before applying the threshold, the digital quantized signal is to be observed properly. A threshold value should then set be accordingly. It is recommended to set the threshold value to a point which is less than half the digital quantized output of the EMG signal. When the digital signal exceeds this threshold, the microcontroller should set an output pin to ' 1 ' and ' 0 ' otherwise [21]. E.g. if the maximum value of the digital quantized signal is 750 (decimal value) then we can set a threshold of 275 . This signal is forwarded to an H-bridge or a motor driver in order to drive the respective electric motors of a robotic mechanism.

The motor driver should be designed or selected according to our requirements of electric motor. Usually a motor driver which can drive a $12 \mathrm{~V}$ motor and handle up to $4 \mathrm{~A}$ current can adequately meet requirements for a robotic arm.

\section{Results and conclusions}

A useful way of acquiring EMG signals and motor drive has been explained in this chapter. Modern microelectronics and controllers have enabled us to develop efficient control of prosthetic robotic mechanisms. To summarize the discussions made earlier, Figure 22 shows a block diagram depicting all the necessary steps required to achieve successful prosthesis. 


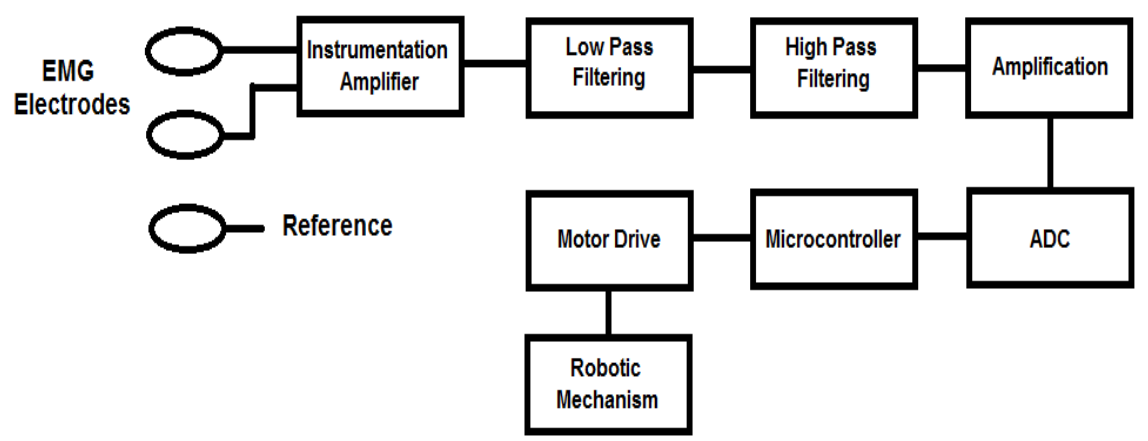

Figure 22. Block diagram indicating all steps for driving a robotic mechanism

As an example, we discuss the control of a robotic hand. There are two primary motions of the human hand, flexing and extending. For flexion, electrode should be placed on Flexor Digitorum Profundus and for extension; the electrode should be placed on Extensor Digitorum Communis [21]. As both muscles exhibit different signal patterns, therefore, a multi-channel input scheme should be employed, so that both signals are gathered independently. Both signals should be observed carefully and a suitable threshold should be set after filtering and amplification. The same procedure is to be followed in order to develop control of all the fingers of the robotic hand i.e. by placing EMG electrodes on specific muscles which control them, allowing us to classify different motions of the hand [22].

The signal observed from a subject with a moderate built is shown in Table 1 . The amplification set for the detected EMG signals from the subject was 10,000. Table 1 provides the EMG signal response from each of the subject's fingers after amplification and threshold set for their control [21].

Size is a very important factor while designing an electronics circuit. A circuit occupying minimum space will be most appropriate in application. A size effective circuit will be easy to place and handle in a robotic mechanism. Advances in biomedical instrumentation have brought fruitful gains to robotic prosthetic technology. The ADS1298 is a 64 pin IC with 8 differential inputs with programmable gain amplifiers (PGAs) and a 24 bit ADC. The PGAs can provide a maximum gain of 12 but the 24 bit ADC quantization scheme is enough to process the EMG signal [23]. With all necessary peripherals attached to a single IC, the size of the whole circuitry can be reduced up to $95 \%$.

Latest robotic researches have enabled us to design and create multi-degree of freedom robotic mechanisms [24]. A good mechanical design and apparatus is essential for efficient robotic prosthesis. Newer electronic components and materials have made robotic prosthesis more functional and adaptable. When we talk about materials, the perfect one should be lighter, durable, adjustable and comfortable for the user. Nowadays, carbon fiber frames are being employed as a solution to this matter. An example of a carbon fiber limb is the state of the art Ottoblock C-Leg. The C-Leg has a built in computer which analyzes data from various sensors and actuates the knee using a hydraulic cylinder. 
When a human uses a robot, he desires to use his natural limb movements to control the mechanism. In order to achieve this, EMG provides the perfect assistance to allow a subject to make normal movements using a robotic apparatus, hence, efficient controllers and improved algorithms are essential for enhanced control of the device. Given the fact that EMG was introduced more than 30 years ago, the research community has a come a long way in coming up with innovative techniques, hardware solutions and advanced procedures to design, control and utilize these signals to produce resourceful prosthetic means to tackle disabilities and amputations effectively.

\begin{tabular}{|c|c|c|c|c|}
\hline S. No. & Finger/ Hand & $\begin{array}{c}\text { Peak Voltage } \\
\text { Reading before } \\
\text { Contracting (V) }\end{array}$ & $\begin{array}{c}\text { Peak Voltage } \\
\text { Reading after } \\
\text { Contracting (V) }\end{array}$ & $\begin{array}{c}\text { Threshold Set } \\
\text { (V) }\end{array}$ \\
\hline 1 & Thumb (flexing) & 0.8 & 3.7 & 1.4 \\
\hline 2 & Index (flexing) & 0.6 & 1.4 & 0.8 \\
\hline 3 & Ring (flexing) & 0.2 & 2.5 & 0.7 \\
\hline 4 & Pinkie (flexing) & 0.2 & 1 & 0.5 \\
\hline 5 & Hand (flexing) & 0.2 & 5 & 1 \\
\hline 6 & Hand (extending) & 0.15 & 4.5 & 0.8 \\
\hline
\end{tabular}

Table 1. EMG signals observed and the threshold in terms of voltage [17]

\section{Future challenges and directions}

Scientists working on upper limb prosthesis define their goal in this field as to develop a 'simultaneous, independent, and proportional control of multiple degrees of freedom with acceptable performance and near "normal" control complexity and response time' [25]. The major challenges faced in prosthetics are: electromechanical implementation, use of EMG control signals and the interface between robotic and clinical communities [26]. Designing a robotic mechanism which is fully capable of integrating with human neuromuscular system is a tough proposition. The requirements can only be fulfilled if the apparatus is of light and flexible material with small but powerful actuators, size effective electronic components, sensors which can easily adapt with the skin and a long lasting battery life. Only then the machine will qualify to be used in everyday practical life [26].

The human hand has 20 degrees of freedom, and the body works in a unique variety of ways to tackle various hindrances placed in front of it. It is therefore, a great challenge to extract all of these motions from the body and utilize them in a resourceful way. Nowadays, two degree of freedom mechanisms are most common. To achieve further DOFs, sensors will be required to be placed at more sophisticated locations, which is a tough task.

The most important challenge of robotic prosthesis in rehabilitation is the feasibility of the mechanism. The apparatus should be comfortable, silent and aesthetically viable for the subject [26]. Our target should be the effective use of the robotic artificial limb on the physically disabled, not to waste our efforts in fruitless objects. Hence, for the reliability of the mechanism's implementation on the amputated population, clinician's approval should be made a part of the procedure. 
Due to its practicality and noninvasiveness, SEMG proves to play a significant role in medical applications and rehabilitation prosthesis. However, the human machine interface will decide if the robotic mechanism will be used in everyday life application or not. It is very important to improve the Quality of Life (QOL) of elder and disabled population. It is believed that in the near future, "we will be able to replace entire limbs with prosthetics that can replicate one's own biological functions precisely, casting natural outward appearance and requiring minimum upkeep" [26].

Robotic researchers and biomedical engineers have been trying to combine their techniques to make the perfect biomechatronic mechanism. However, in order to ensure that challenges are met and to create a more smart and intelligent machine, communication between clinicians, users and engineers should be established on a greater scale.

\section{Author details}

Muhammad Zahak Jamal

National University of Sciences and Technology, Pakistan

\section{Acknowledgement}

The study was carried out at College of Electrical and Mechanical Engineering (CEME), NUST in collaboration with Armed Forces Institute of Rehabilitation Medicine (AFIRM). The author is highly indebted to Brig. Dr. Javaid Iqbal and Dr. Umer Shahbaz Khan for their help in the study and CEME for providing necessary funds to make this research possible. Special thanks to all colleagues and people who have willingly helped out with their abilities.

\section{References}

[1] Alan G. Outten, Stephen J. Roberts and Maria J. Stokes (1996) “Analysis of human muscle activity", Artificial Intelligence Methods for Biomedical Data Processing, IEE Colloquium, London

[2] Musslih LA. Harba and Goh Eng Chee (2002) "Muscle Mechanomyographic and Electromyographic Signals Compared with Reference to Action Potential Average Propagation Velocity", Engineering in Medicine and Biology Society, 19th Annual International Conference of the IEEE, Vol.3

[3] Nissan Kunju, Neelesh Kumar, Dinesh Pankaj, Aseem Dhawan, Amod Kumar (2009) "EMG Signal Analysis for Identifying Walking Patterns of Normal Healthy Individuals" Indian Journal of Biomechanics: Special Issue

[4] Carlo J. De Luca (1997) "Use of Surface Electromyography in Biomechanics" Journal of Applied Biomechanics, Vol.3

[5] Carlo J. De Luca (2006) “Electromyography: Encyclopedia of Medical Devices and Instrumentation" (John G. Webster Ed.), John Wiley Publisher

[6] Jarret Smith (2010) image title: "motor-unit-lg" 
[7] S.L. Pullman, D.S. Goodin, A.I. Marquinez, S. Tabbal and M. Rubin (2000) "Clinical Utility of Surface EMG" Report of the Therapeutics and Technology Assessment, Subcommittee of the American Academy of Neurology, Neurologly Vol. 55:171-177

[8] Paul E. Barkhaus and Sanjeev D. Nandedkar (2000) "Electronic Atlas of Electromyographic Waveforms" Vol. 2, 2 ${ }^{\text {nd }}$ Edition

[9] Nuria Masso, Ferran Rey, Dani Romero, Gabriel Gual, Lluis Costa and Ana German (2010) "Surface Electromyography and Applications in Sport" Apunts Medicina De L'Esport, Vol. 45: 127-136

[10] Dr. Scott Day "Important Factors in Surface EMG Measurement", Bortec Biomedical Incorporated

[11] (2008) "Bagnoli EMG Systems Users Guide", Delsys Incorporated

[12] Netter FH (1997) “Atlas of Human Anatomy" East Hanover, New Jersey: Novartis.

[13] Elaine Marieb and Katja Hoehn (2007) "Human Anatomy and Physiology" $7^{\text {th }}$ Edition, Pearson Education

[14] Björn Gerdle, Stefan Karlsson, Scott Day and Mats Djupsjöbacka (1999) "Acquisition, Processing and Analysis of the Surface Electromyogram". In: U. Windhorst, H. Johansson, editors. "Modern Techniques in Neuroscience Research", Springer

[15] Carlo J. De Luca (2002) "Surface Electromyography: Detection and Recording", Delsys Incorporated

[16] D.J. Hewson, J.Y. Hoqrel and J. Duchene (2003) "Evolution in impedance at the electrode-skin interface of two types of surface EMG electrodes during long-term recordings" Journal of Electromyography and Kinesiology, Vol. 13, Issue 3 , pp. 273-279

[17] (2009) "Instrumentation Amplifier Application Note", Intersil Incorporated

[18] Gianluca De Luca (2001) “Fundamental Concepts in EMG Signal Acquisition”, Delsys Incorporated

[19] P.R.S. Sanches, A.F. Müller, L. Carro, A.A. Susin, P. Nohama (2007) "Analog Reconfigurable Technologies for EMG Signal Processing" Journal of Biomedical Engineering, Vol. 23, pp. 153-157

[20] M. E. Van Valkenburg (1982) “Analog Filter Design”, Holt, Rinehart \& Winston

[21] Zahak Jamal, Asim Waris, Shaheryar Nazir, Shahryar Khan, Javaid Iqbal, Adnan Masood and Umar Shahbaz (2011) "Motor Drive using Electromyography for Flexion and Extension of Finger and Hand Muscles" $4^{\text {th }}$ International Conference on Biomedical Engineering and Informatics, Vol. 3 pp. 1287-1291

[22] Sebastian Maier and Patrick van der Smagt (2008) "Surface EMG suffices to classify motion of each finger independently" Proceedings of MOVIC 2008, 9th International Conference on Motion and Vibration Control

[23] Datasheet ADS1298 “Low-Power, 8-Channel, 24-Bit Analog Front-End for Biopotential Measurements" Texas Instruments Incorporated.

[24] A. H. Arieta, R. Katoh, H. Yokoi and Y. Wenwai (2006) “Development of a Multi D.O.F Electromyography Prosthetic System Using Adaptive Joint Mechanism", Applied Bionics and Biomechanics, Vol. 3, Woodheads Publishing

[25] D. Edeer and C.W. Martin (2011) “Upper Limb Prostheses - A Review of the Literature with a Focus on Myoelectric Hands", WorksafeBC Evidence-Based Practice Group

[26] Brian Dellon and Yoki Matsuoka (2007) "Prosthetics, Exoskeletons, and RehabilitationNow and the Future" IEEE Robotics \& Automation Magazine, March, 2007 